\title{
My Naturewatch Camera
}

\section{Disseminating Practice Research with a Cheap and Easy DIY Design}

\author{
William Gaver \\ Interaction Research Studio \\ Goldsmiths, University of London \\ London, UK \\ w.gaver@gold.ac.uk \\ Andy Sheen \\ Interaction Research Studio \\ Goldsmiths, University of London \\ London, UK \\ a.sheen@gold.ac.uk \\ Naho Matsuda \\ Interaction Research Studio \\ Goldsmiths, University of London \\ London, UK \\ n.matsuda@gold.ac.uk
}

\author{
Andy Boucher \\ Interaction Research Studio \\ Goldsmiths, University of London \\ London, UK \\ a.boucher@gold.ac.uk \\ Dean Brown \\ Interaction Research Studio \\ Goldsmiths, University of London \\ London, UK \\ d.brown@gold.ac.uk \\ Amina Abbas-Nazari \\ Design Products \\ Royal College of Art \\ London, UK \\ amina.abbas-nazari@rca.ac.uk
}

\author{
Michail Vanis \\ Interaction Research Studio \\ Goldsmiths, University of London \\ London, UK \\ m.vanis@gold.ac.uk \\ Liliana Ovalle \\ Interaction Research Studio \\ Goldsmiths, University of London \\ London, UK \\ l.ovalle@gold.ac.uk

Robert Phillips
Design Products
Royal College of Art
London, UK
robert.phillips@rca.ac.uk

\begin{abstract}
My Naturewatch Camera is an inexpensive wildlife camera that we designed for people to make themselves as a way of promoting engagement with nature and digital making. We aligned its development to the interests of the BBC's Natural History Unit as part of an orchestrated engagement strategy also involving our project website and outreach to social media. Since June 2018, when the BBC featured the camera on one of their Springwatch 2018 broadcasts, over 1000 My Naturewatch Cameras have been constructed using instructions and software from our project website and commercially available components, without direct contact with our studio. In this paper, we describe the project and outcomes with a focus on its success in promoting engagement with nature, engagement with digital making, and the effectiveness of this strategy for sharing research products outside traditional commercial channels.
\end{abstract}

Permission to make digital or hard copies of all or part of this work for personal or classroom use is granted without fee provided that copies are not made or distributed for profit or commercial advantage and that copies bear this notice and the full citation on the first page. Copyrights for components of this work owned by others than the author(s) must be honored. Abstracting with credit is permitted. To copy otherwise, or republish, to post on servers or to redistribute to lists, requires prior specific permission and/or a fee. Request permissions from permissions@acm.org. CHI 2019, May 4-9, 2019, Glasgow, Scotland UK

(c) 2019 Copyright held by the owner/author(s). Publication rights licensed to ACM.

ACM ISBN 978-1-4503-5970-2/19/05 ..\$15.00

https://doi.org/10.1145/3290605.3300532

\section{CCS CONCEPTS}

- Human-centered computing $\rightarrow$ Empirical studies in interaction design.

\section{KEYWORDS}

Design research; research through design; DIY design; opensource product; camera; nature; wildlife

\section{ACM Reference Format:}

William Gaver, Andy Boucher, Michail Vanis, Andy Sheen, Dean Brown, Liliana Ovalle, Naho Matsuda, Amina Abbas-Nazari, and Robert Phillips. 2019. My Naturewatch Camera: Disseminating Practice Research with a Cheap and Easy DIY Design. In CHI Conference on Human Factors in Computing Systems Proceedings (CHI 2019), May 4-9, 2019, Glasgow, Scotland UK. ACM, New York, NY, USA, 13 pages. https://doi.org/10.1145/3290605.3300532

\section{PRELUDE}

Chris Packham, naturalist, author and presenter of BBC's Springwatch series, walks beside Bill Gaver towards set of unusual looking devices in a leafy clearing. To the side the ribbed metal wall of an academic building can be glimpsed, offering an incongruous frame to the scene.

"Bill, you've got yourself a little grotto here, full of - how shall I put it - very homemade gadgetry. What's this - this is a camera is it?"

"That's right, that's a camera trap. And it's meant to look homemade because we want people making them at home. It's all off the shelf parts, it's really cheap-it costs people about £25 to make this." 


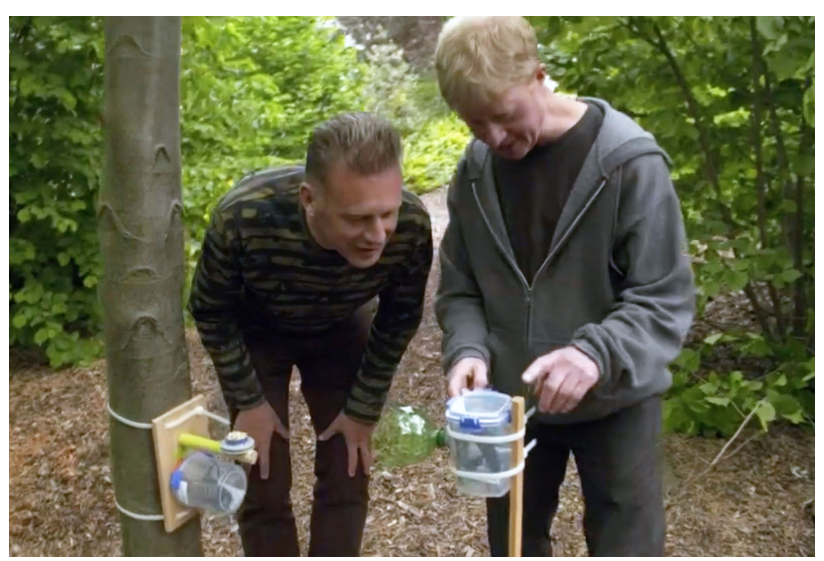

Figure 1: Springwatch presenter Chris Packham discusses My Naturewatch Camera with Bill Gaver.

"I love the recycling element to it, and this is obviously something straight out of the kitchen."

"That's just a food storage container, works really well, it's super easy to make."

"It's dead cheap, you're recycling, I love all that. I could put that together myself in my office. But does it work, Bill?"

"Oh yeah." Gaver pulls out his phone and shows Packham a photograph of a squirrel.

"I've got to say, that's actually pretty good."

\section{INTRODUCTION}

My Naturewatch Camera is a simple digital camera designed to be positioned outdoors, usually in front of bait (Figure 1). It uses computer vision software to look for movement and trigger image capture, and thus can automatically take photographs of birds and animals that come in view without human presence. We designed it to be used in people's gardens or local green spaces to record images of nearby wildlife.

The cameras are available as DIY designs for people to make themselves. Constructed entirely from commercially available components, using instructions and software from our project website (mynaturewatch.net), they are designed to be as easy to make as possible, and to require no direct contact with the project team for success.

We designed My Naturewatch Camera as part of a project that we initiated when we realized that, by virtue of its appeal to a large audience, the Springwatch series could be a key component of an orchestrated strategy for promoting large-scale engagement with research products by encouraging people to make DIY devices designed to promote engagement with digital making and local wildlife. We unpack each of these themes (dissemination, DIY designs and engagement) in the paper's discussion section. For now, it is enough to say that, as an initiative integrating these concerns, the project makes two primary contributions: 1 ) the design of an innovative, self-built wildlife camera-a research product [32] investigating how computational devices can promote engagement with making and nature-and 2) an example of an orchestrated strategy bringing together a national primetime TV show, instructional website, and social media outreach to encourage people to make the camera. In the following account, these themes and contributions underlie our account of the development and dissemination of the devices.

\section{Springwatch: Setting and Vehicle}

The Springwatch series, and its spin-offs Autumnwatch and Winterwatch, are world-leading examples of public engagement with science. Springwatch is produced by the Natural History Unit (NHU), a BBC department that has been dedicated to producing wildlife content since 1957 and which is responsible for major series such as Planet Earth and The Blue Planet.

Springwatch is usually broadcast over an intensive threeweek period, live from a temporary encampment usually in a nature reserve. The core of the show consists of footage gathered from numerous outdoor cameras that film birds and animals throughout the site, as well as footage from other locations, all strung together, enlivened and enriched with commentary from experts, particularly Chris Packham, who has been the lead presenter since 2009. Several TV shows are broadcast daily, with further content available via the BBC 'red button' digital interactive television service as well as a series website, allowing viewers to immerse themselves in the stories of individual animals as well as features on issues including flooding or global warming. With UK daily viewing figures of about 2 million, Springwatch engages an impressive number of people with its mix of animal soap operas and detailed information.

With its balance of entertainment and expertise, Springwatch seemed well-suited as a frame for research devices that would be engaging but not utilitarian (i.e., they wouldn't contribute to Citizen Science, at least as narrowly conceived). We hoped that such devices would be of interest to Springwatch as a complement to the programme's usual format as a broadcast from a small production team to a large audience by allowing audience members themselves to engage with nature locally. Finally, we anticipated that exposure on Springwatch would motivate some of the show's audience to make the DIY designs, thus allowing our research products to circulate to a wide and diverse public.

\section{A Delicate Relationship}

We initially contacted the NHU, before applying for project funding, via a friend at the BBC who introduced us to a longterm consultant to the unit. She responded enthusiastically to our ideas and invited us to visit the Springwatch set. She 
introduced us to a few members of the team, including their Business and Communications Manager, who eventually wrote a guarded letter of support for our funding application that was, no doubt, pivotal to its success.

By the time we had secured project funding, however, about half a year had passed since our previous contact, and our project had largely been forgotten. When we finally met about half a dozen members of the Springwatch team-including the series producer-to discuss our plans, we essentially had to pitch the project afresh. The nature of our funding allowed us to make a compelling offer:

- We would develop technologies for engaging with wildlife that viewers could make at home;

- the devices and our time would not cost the BBC anything;

- they were under no obligation to broadcast the designs or, indeed, to engage with the project;

- all we asked was for them to meet with us occasionally to see if our ideas were of interest.

This characterisation of the project (essentially we would give them something for nothing and they didn't have to take it) appeared reassuring to the members of the NHU, and set the tone for our work throughout our development of the My Naturewatch camera. It implied, and we encouraged, a lack of commitment from the BBC, to the point that, even after they had filmed the feature described at the opening of this paper, they stressed that they could not be sure to air it until the moment it was used in their live broadcast. The uncertainty this engendered made the project risky for us, of course, and was uncomfortable at times. However, it was also, we believe, vital to the congenial relationship we formed with the Springwatch team-a relationship better characterised as a constantly negotiated alignment of our project to their interests rather than an established collaboration-allowing them to discuss speculative, implausible and even humorous ideas without risk.

\section{OPENING A DESIGN SPACE}

To the opening meeting, we also brought a design workbook [13] we had developed over the first several months of the project and discussed it with the group.

The workbook thematically organised a set of 64 sketch proposals-simple juxtapositions of images with short captions or titles indicating a direction our designs might take (Figure 2)-that we had developed based on our background research as well as the visit to the Springwatch set. The proposals opened a wide space of possibilities with various degrees of complication and playfulness.

Discussing the workbook provided the Springwatch team with something of a crash course to our conceptual and

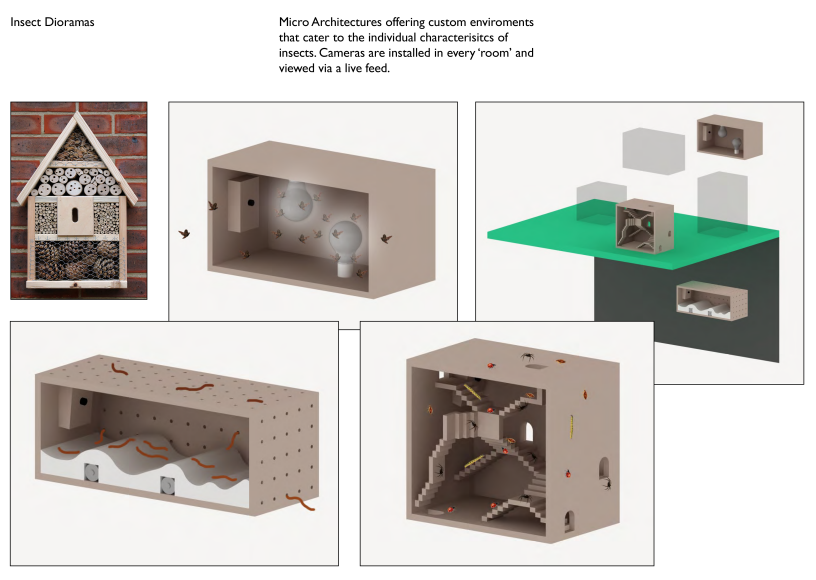

Figure 2: Sample proposal: specialized habitats for filming microbeasts. $\odot$ Interaction Research Studio

aesthetic practice as well as the ideas we were pursuing. Fortunately, they were receptive, and our discussion was lively and productive. This set the tone for a cycle of engagement over the next six months in which we modified, developed and came up with new proposals in our studio, based on discussions we had during two further meetings with the Springwatch team.

Over this time, we gained a sense of the qualities of our designs that would make them attractive for Springwatch. Fundamentally, they needed to match the skillful balance achieved by the programme itself between conveying knowledge-based content and (always respectful) entertainment. Achieving a similar balance with our designs would be essential to appeal both to Springwatch and its audience. It was also desirable that the devices be maximally accessible, both in terms of the technologies and skills needed to construct them, and their intended uses. For instance, devices designed to interact with foxes or badgers would exclude those without large mammals nearby, while focusing on 'microbeasts' (e.g. insects, slugs, worms etc.) would be accessible to most viewers. Finally, we were told that, to be suitable for Springwatch, our designs needed to be relevant for an 'editorial line'-a somewhat mysterious concept that we understand to mean that the devices must serve as resources for stories with content the team was interested in covering.

\section{Focusing on a Wildlife Camera}

As our work progressed, then, we moved away from overly specific or complicated proposals in favour of simpler ones that might develop over time. In particular, we started concentrating on the possibility of building a DIY wildlife camera because this, we believed, would be relatively simple to make, could be used in settings from windowsills to wilderness, and would capture images of wildlife from snails to 
sealions. Moreover, a large number of our existing proposals integrated cameras into more complicated designs (such as animal tracking or nationwide events). If enough people made their own wildlife cameras, we envisioned the possibility of suggesting complementary designs or events that would particularise their use.

Researchers have used automatic wildlife cameras (a.k.a. camera traps or trail cams) for decades, and they have become increasingly popular commercially as well, particularly among hunters wanting to know where to find game [44]. From the outset, however, we intended our version to be different from commercial ones in two ways. First, ours would be designed for nonutilitarian and aesthetic uses, not for scientific research or hunting. Second, they would be designed for people to build themselves, cheaply and easily.

The Naturebytes research project [30] also distributes a wildlife camera for people to build, but it comes in kit form, and essentially recreates the features of standard commercial versions. Our experiences using a number of commercial camera traps that we purchased-including a Naturebytes one-convinced us that we could design a wildlife camera that would not only be easier to build and more suited for playful experimentation, but also offer improvements over the other types of camera trap we had encountered. Most notably:

- Relying on IR sensing leads to many false positives when pictures are triggered and is very poor at detecting small animals and especially birds. We resolved to use computer vision to achieve motion detection instead.

- Many versions incorporate IR light sources for night time illumination. Because these tend to be placed immediately adjacent to the camera lens, they lead to 'surveillance style' portraits of animals (Figure 3).

- The versions we used had screens and controls incorporated inside their waterproof housings, making access for positioning, controlling and retrieving images awkward and inconvenient.

As we developed our design for a DIY wildlife camera, we sought to address these shortcomings. Initially we experimented with stringing off-the-shelf apps together to trigger a smartphone to take photographs based on motion, and used this to create an 'underwater camera'-essentially a phone in a weighted mason jar-which we displayed, along with instructions for how to make it, in a London design exhibition [8]. Based on this experience, we chose the hardware and developed the software for a user-made, stand-alone version. Multiple iterations of the camera followed as we tested it in workshops held in several nature reserves, schools and colleges, before we finalised the My Naturewatch Camera design described below.

\section{MY NATUREWATCH CAMERA}

The final design for our DIY wildlife cameras is a configuration of computational hardware, software, and strategies for making weather-resistant housings. It should be stressed that the design is completely open-sourced, and thus different hardware can (and has) been used, the software can be (and has been) modified, and wide variety of housings can be (and have been) made. Our description here is for the version described by our website and featured on Springwatch 2018.

\section{Hardware}

My Naturewatch Camera is designed to be implemented on the Raspberry Pi Zero W microprocessor platform, an inexpensive $\sim \$ 10$ USD) single-board computer. Apart from its price and power, two features make it ideal for the cameras: first, it includes a built-in graphics processing unit and can make use of Unix computer vision libraries; second, it also includes WiFi capabilities allowing it to be connected to and controlled by external devices (i.e. smartphones, tablets and laptops). Finally, it includes a microSD slot allowing its software to be read from, and the pictures it takes to be written to, a standard microSD card.

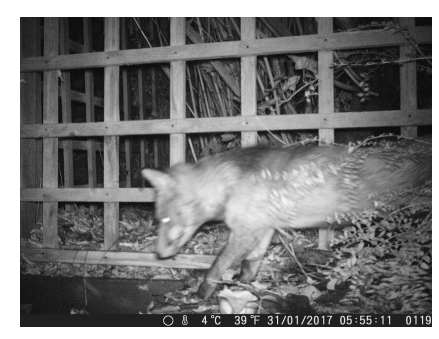

We use a 'standard' camera module for the Raspberry Pi Zero, a small (roughly $1 \mathrm{~cm}^{2}$ ) unit mounted on a flexible circuit board with a ribbon cable that slots directly into the Raspberry $\mathrm{Pi}$. The unit is inexpensive $(\sim £ 14)$, provides a good quality (5MP) image, and is

Figure 3: 'Surveillance style' image from a commercial camera trap. Raspberry Pi Zero, however, allowing a variety of options. For instance, we also provide instructions on our website for an infrared version, using a unit that incorporates infrared lights as well as an unfiltered camera. Advanced makers can modify this, with a modicum of soldering, by separating the lights from the cameras to avoid 'surveillance style' images.

The processor on the Raspberry Pi Zero W can become hot when used intensively (for instance to process incoming images), so we recommend adding a low-tech heat sink in the form of a standard flat-headed bolt (about M6 x 25mm) superglued to the processor. The final device can be powered with an off-the-shelf USB powerbank (ideally over $12000 \mathrm{mAh}$ in capacity) and will run for about eight hours depending on the powerbank and number of pictures taken. 

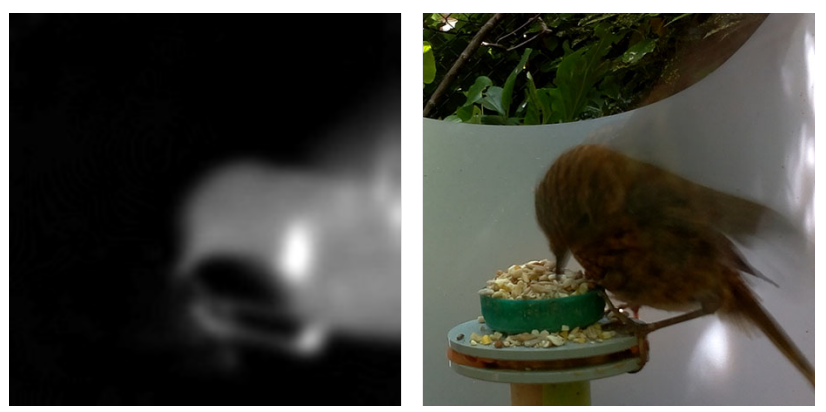

Figure 4: A 'difference image' (left) triggers image capture a moment later (right) when contiguous areas of change are within a minimum and maximum threshold.

\section{Software}

Software for My Naturewatch Camera is downloaded from the website and installed on a microSD card for use by the Raspberry Pi.

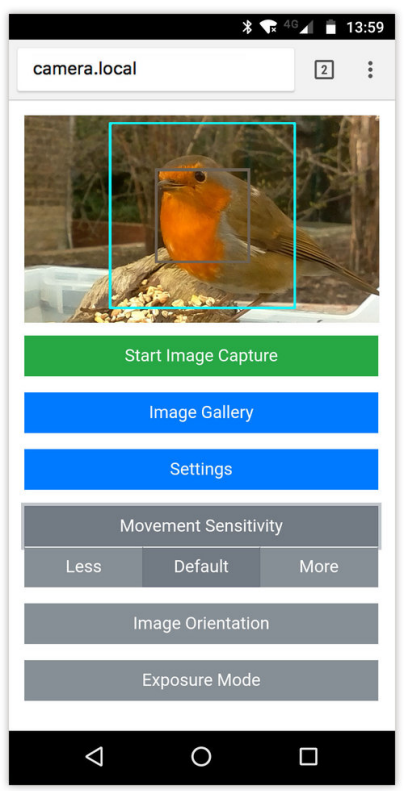

Figure 5: Camera web controls on a smartphone.

group them into larger on group them into larger one contour in the result is within a given threshold. This helps reduce the false positives we observed in IR triggered camera traps: minimum and maximum thresholds enable the camera to ignore overall changes in lighting or small movements.

My Naturewatch Camera creates a local wifi hotspot and serves a webpage (Figure 5) allowing people to control it via their own devices (e.g. tablet, smartphone, laptop). This enables the device to be used away from existing networks, and allows the image captured by the camera to be viewed live, facilitating placement, before starting automatic image capture. In addition, it allows changing the camera settings via a dropdown menu-for instance, the minimum and maximum amount of change needed to trigger capture, indicated by coloured squares drawn on the image, can be controlled via the 'movement sensitivity' buttons. Selecting the 'Image Gallery' button serves a new page showing all the images held on the camera's SD cards and allows pictures to be deleted or downloaded to the connected device.

Most of the controls for the camera are provided by its internally served webpage, however a configuration file on the SD card allows a few other settings, including the camera's name and password, to be set separately.

\section{Housing the Camera}

For use outdoors, it is wise to house the My Naturewatch Camera hardware to protect it from the elements. This can be done in innumerable ways-we have enclosed them in resealable zipper bags, while others have constructed elaborate $3 \mathrm{D}$ printed housings. Perhaps the most im-

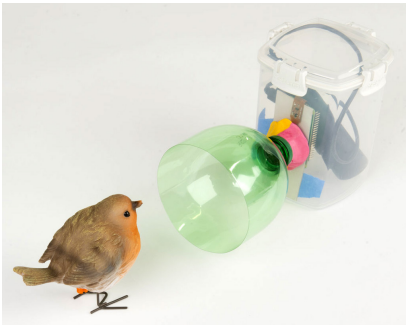

Figure 6: Typical My Naturewatch Camera housing. portant lessons we have learned is that for clarity of image it is best not to enclose the camera lens, and that, fortuitously, the hardware is remarkably resistant to water damage.

Our standard approach is to house the camera electronics in a waterproof plastic food storage container with a hole drilled in to expose the camera lens, and to shield the lens from rain with the cut-off end of a plastic bottle (Figure 6). Full instructions for making this housing is on our website. Apart from the fact that it is easy to make, this approach emphasises the accessibility of the camera, both by appearing easy to make, and by exposing the electronic components within (c.f. [43]).

\section{MyNaturewatch.net}

A final, and crucial, component of the My Naturewatch Camera configuration is the project website. For most people, this is the only contact point with the project and the sole source of information about My Naturewatch. It is essential in motivating people to make the cameras, enabling them to do so, and allowing them to discuss problems and experiences. Three main sections are fundamental to the website's operation. 
Make Your Camera provides instructions for constructing the standard daylight My Naturewatch Camera. These are presented in an illustrated 'recipe' format similar to one developed for the Interaction Research Studio's ProbeTools project [5] and explored independently in [9]. A list of parts and recommended tools, most linked to online suppliers, is at the top of the page. This is followed by an outline summary of the build process, and then step-by-step instructions for making the camera, separated into basic tasks (e.g. "Download the Software", "Assemble the Electronics", etc.). Each step is accompanied by photos or short videos of the build to make the process clear; working through all the steps produces a finished and working My Naturewatch Camera.

A link from the home page, and another from the end of the 'Make' page, leads to a Using Your Camera page. This provides step-by-step illustrated instructions for the camera's interface, showing how to preview images, start automatic recording, download photos, etc. This page also provides a few tips for using the camera successfully and ethically.

Finally, a Forum page leads to a user forum allowing messages and responses to be entered by registered users, organized according to categories that we established on set-up ('Technical Issues', 'Camera Builds and Set Ups', etc.). We created the forum about two weeks after the Springwatch episode aired, after finding ourselves overwhelmed by the demands of an alternative support system that required individual responses. The Forum is a busy and valuable area of the website that we discuss later.

\section{RELEASING MY NATUREWATCH CAMERA}

My Naturewatch Camera featured in the Springwatch episode broadcast on 11 June 2018, as part of a 10-minute-long feature $^{1}$ seen by an audience of just under 2,000,000 [4]. The coverage included footage from a visit to the Goldsmiths campus a month earlier, discussions between the presenters on the Springwatch set and a segment in which another Springwatch presenter deploys the camera on site in an (unsuccessful) attempt to photograph a local fox. On the same day, the camera was featured on Wild Academy, a spin-off of Springwatch targeted at school children. On both programmes, the camera was featured as a device designed for viewers to make at home.

Response was immediate. The evening of the show, mynaturewatch.net attracted 5,999 unique visitors, with 15,766 visiting over the following week, and a total of 21,867 at the time of writing-about $1 \%$ of the audience.

In the following sections we discuss what we learned about the people who made their own My Naturewatch Cameras.

\footnotetext{
${ }^{1}$ The segment also included another project design that we plan to report separately in the future.
}

\section{How Many People Made My Naturewatch Cameras?}

It is impossible to know precisely how many people have made My Naturewatch Cameras. Because they are based on commercially available hardware that can be purchased from multiple sources, we can only estimate based on other sources of information.

One clue comes from Pimironi.com, a hobbyist electronics company that sold all the components needed to build a My Naturewatch Camera as a 'bundle' on its website, which we linked from the Make Your Camera section of ours. As of the time of writing, they had sold about 1,050 bundles [Todd, personal communication]. People could also purchase components individually, however, and some used equipment they already owned, so this is almost certainly an underestimate.

A better indication is the number of downloads of the camera software from our website. To date there has been a total of 1,086 downloads from unique IP addresses of the first software version (v 0.2.5), and 389 (v 0.4.2) of the second. Assuming some but not all are upgrades of cameras that people already built, that would indicate that between 1,086 and 1,475 people have built the camera. We expect the truth to be at the high end of this scale, both because there are few reports of people upgrading their software and because many people report making multiple cameras. All in all, then, we're confident that between 1,250 and 1,500 cameras have been made to date.

\section{Glimpses of Use}

We strove to minimise any barriers to making My Naturewatch Camera, so for instance did not require people to register to download the camera software. Since that was the only mandatory touchpoint to the project, anonymous participation was default and we have little or no access to the vast majority of people who made the cameras.

We did, however, encourage people to post pictures and feedback on social media such as Instagram and Twitter. In addition, 76 people posted reviews on the Pimoroni website, and many of these comment on the cameras as well as Pimoroni's service. Finally, 158 joined the mynaturewatch forum to post comments, pictures and (especially) requests for help. The observations in the following sections thus draw on these sources ${ }^{2}$.

\section{Overall Satisfaction}

In general, people who made the cameras seemed pleased with the experience. This was especially evident from the

\footnotetext{
${ }^{2}$ Note that mynaturewatch.net/forum, shop.pimoroni.com, and twitter.com/mynaturewatch are all open to the public, so readers may view comments and examples of practice for themselves.
} 
comments made on the Pimoroni website. A typical example is from Richard:

A great little project and really fun to use. Took me a few minutes to assemble, maybe an hour to download and write the new flash image. Couldn't wait to get it working in the garden and immediately I got a lovely picture of goldcrests bathing in my pond.

These sentiments were echoed by many other comments. Martin wrote: Simply Brilliant. An absolute dream. Phillip commented: I'll be honest. I wasn't expecting such great results so quickly. From David: Awesome piece of kit very easy to set up make, I love it that much I've just ordered myself another full kit. Jeremy's contribution: the pictures that my son managed to get were as he said "brill" yet another happy child. Many of the comments continue in this vein, with the camera kit scoring over 4.5 out of 5 stars on the website.

\section{Inexperienced Makers}

Reading the comments, it becomes clear that many of the people who made My Naturewatch Cameras were technically inexperienced. For instance, an anonymous comment on the Pimoroni site:

I was a little nervous of trying this kit as $i$ am not the most techy geek in town. However it was easy to make, set up and use. The results are great and we will try it in lots of different places. So far got pictures of black birds and robins mostly. Interesting how often they have their backs turned to the camera!

Another anonymous comment: Impressive quality of pictures and fairly easy to set up even for someone like me who's not very technically minded. Mike commented that the camera was easy to set to work by an amateur like me. Finally, another anonymous Pimoroni client wrote:

Was not sure about this as never put together anything like it before and wondered if it would actually work. Followed the instructions which were pretty straightforward and it worked first time. Very pleased with it.

Comments like these suggest that presenting My Naturewatch Camera on Springwatch succeeded in attracting a technically inexperienced audience, and that the simple design of the camera itself facilitated their successful engagement with digital making.

\section{Tailoring My Naturewatch Cameras}

There are also numerous reports on social media and our forum of people tailoring their My Naturewatch Cameras.
Most are variations of the housing suggested on our website (Figure 6). Several people found simple take-away boxes easier to modify than the clip-top ones we recommended. PrioryJim discovered that the bottle could be attached to the food storage container with its own cap, after drilling a hole for the camera to see through. Michael, an ornithologist, protected the camera hardware in a zipped plastic bag before hanging it in a tree to view a buzzard's nest. Hc25036 sent pictures of a more elaborate build in which he draped the camera cable over a wooden partition to peer into a hedgehog feeding station. In general, the camera design permitted many variations.

Other camera makers described more sophisticated technical modifications, revealing their expertise. For instance, forum user Ricko352 wrote I soldered a couple of pins to header $4 \& 6$ (+5v \& gnd) so that I could add a fan for circulating air over the Raspberry Pi Zero $W$ board. A Pimoroni, employee, gadgetold, reported a modification to:

... add hardware buttons for arm/disarm and snap

photo. I used a Button SHIM for this since I'm

somewhat biased toward it, and I already had a

Zero $W$ with one soldered in place...

Still others modified the camera software, which we have made available as a GitHub project. So far, four of the suggested changes have been incorporated into the latest version of the software we release. For instance, one contributor's changes formed the foundation for error-checking code which allows the camera to be un- and re-plugged without crashing. In general, the variety of modifications people have reported indicate both the range of expertise that My Naturewatch Camera makers bring to the project, and the flexibility of the camera to be modified in many ways depending on that expertise.

\section{Importance of the Forum}

We established the mynaturewatch.net forum as a way for people to ask for help making the devices, and although we also added other topics, most of the discussion has revolved around difficulties people have had making the kit. There are 158 forum members (10 - 15\% of makers), 164 topics, and 987 replies. The majority (91 topics / 897 posts) are to the 'Technical Issues' section with 69 topics / 250 posts spread across 'Using the My Naturewatch Camera', 'Camera Builds and Setups', and 'Project Feedback'. Common problems have to do with downloading the software and installing it on the SD card, or providing adequate power to the camera, or focusing the camera to achieve sharp pictures. These are well-understood problems and can usually be rectified fairly quickly. 
A more serious problem arose, however: The UK entered an unusually hot summer soon after the cameras were released, and we received a number of reports of the cameras halting which were eventually traced to overheating. In an intense exchange over several weeks, a number of forum members reported temperatures they had recorded from the Raspberry Pi and numerous fixes ranging from various heatsinks to added fans and even a Peltier cell. A satisfactory resolution, however, only came when we released a new version of the software which, among other things, dramatically reduced demand on the CPU-and the advent of cooler weather.

Some users became quite irate when they could not make their cameras work, often blaming the technology or design. For instance, Bullstreetboy wrote, under the header This project does not work reliably:

Guys

I give up.

I've wasted countless hours. I've changed hardware, software, SD cards, Power banks, Pi zeros EVERYTHING.

...From a great start to a shambles.

Usually even these people managed to get their cameras working in the end, however. For example, after much struggle, Bullstreetboy finally solved his problems, which he announced with a simple (and rather poetic) message:

Problem solved

Power bank to PI cable
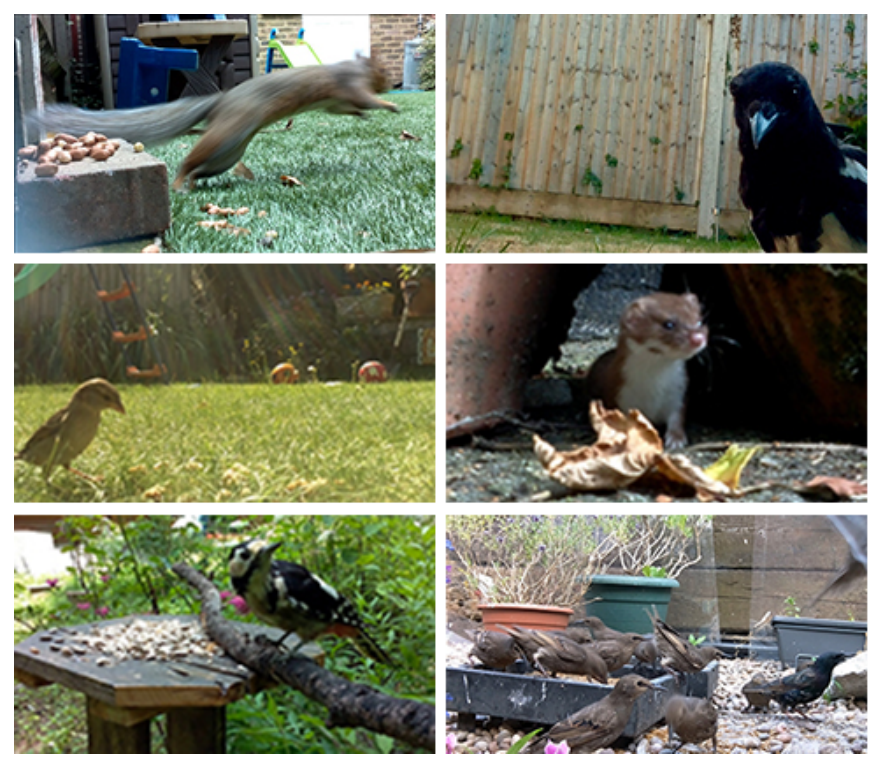

In fact, Bullstreetboy went on to become a frequent and helpful contributor to the forum, making helpful suggestions to other members and reporting that he was starting to build a second, infrared camera-before again becoming incensed when he could not replicate his original success.

Over time we found that we could reduce our involvement with the forum as new members looked at existing posts to find answers to their problems, and existing members helped them with those they could not solve themselves. The forum community thus became an invaluable resource in supporting the large numbers of people, many with little technical experience, who made My Naturewatch Cameras.

\section{Photographs and Appreciation}

Since the Springwatch broadcast, hundreds of My Naturewatch Camera photographs have been posted to our forum and to social media sites such as Twitter (Figure 7). It is evident from comments on the forum and social media that these are a tiny fraction of the pictures that have been taken: it is not unusual for hundreds of pictures to be taken in a single session, most containing animals.

Photographs have included foxes, badgers, weasels, deer, mice, rats and hedgehogs, and these animals are greeted by appreciative comments from other My Naturewatch Camera makers, presumably because they are only occasionally seen in everyday life (squirrels, in contrast, are usually remarked upon for their mischievousness). By far the majority of photographs, however, are of birds: robins, blackbirds, goldfinches, woodpeckers, buzzards and many, many pigeons. Perhaps surprisingly, these too are a source of pride to

Figure 7: Pictures taken with My Naturewatch Cameras. Credits from left to right, top to bottom: @mrcjbush, @keirwhitlock, Leila Racaniere, dcurzon, tattyjacket, @WheatlandFarm, roadkillcrow, Ricko352, @nariyasu, alanr00, stewartL, @ISpaceCab 
their authors, and attract complements from other viewers, despite their ubiquity in the British landscape. We speculate that there may be two reasons for this.

First, photographs captured by My Naturewatch Cameras tend to exhibit characteristic aesthetic qualities. Their view is often at the animal's level and from relatively short distances. In addition, they often have a kind of candid quality, as animals are captured unawares. The result is a kind of intimacy in the views they afford. Second, for their camera owners, the photographs are of the animals in their own local surroundings. They are not generic pictures of birds or animals, but portraits of the individuals that live in their gardens: their neighbours. This, we believe, lends both the photographs and the animals themselves a personal value to people. It is fundamental to their appreciation of My Naturewatch Cameras, and, we believe, to the cameras' success in promoting increased engagement with nature.

\section{A Final Quote}

Carole's contribution to our web forum might serve as the best summary of our findings:

I use a laptop, iPad and phone but that's it, I just use them. This looked interesting and there are several places I know animals travel along in my garden and I thought it would be nice to find out more about them.

I needed help with the zip file as I had no idea how to deal with it but building the camera and box was pretty straightforward.I got the camera upside down to start with, had a bit of a struggle fitting everything neatly into my box and superglued my fingers together, all in all a learning experience but easier than building flatpack furniture.

The overheating issues were a challenge, but this discussion group has helped. Now that appears to be resolved for me I am having fun, not many successes yet but I look forward to finding out what is in my compost heap (hopefully not rats) and if it is only rabbits using the hedgehog runs under the fence.

I may even move on to an IRcamera to see if the foxes that passed through the other day visit more often.

These are all things I would not have attempted without Naturewatch - many thanks to you all.

Not surprisingly, we have been pleased to receive comments such as these-and all the more so when Carole finally posted a picture of a rabbit in her garden (Figure 8).

\section{DISCUSSION}

My Naturewatch Camera is a DIY design that has been made and enjoyed by a wide range of people. Here we discuss the project in terms of the three themes that inspired it: engagement with nature, digital making, and the circulation of research products, before summarising our contributions in the conclusion.

\section{Engagement with Nature}

In offering engagement with local wildlife, My Naturewatch Cameras can be seen as an antidote to the ways that popular media often dramatise 'nature' as exotic, alien or dangerous, whether on the Discovery Channel's 'Shark Week' [8] or the BBC's Blue Planet II [2], with its stunning depictions of unseen species and unusual behaviours. Even Springwatch tends to feature spectacular photography from UK nature reserves, land explicitly set apart from everyday living environments. My Naturewatch cameras can be seen as complementing these typical representations with more quotidian content that people can capture themselves.

Despite burgeoning interest in Animal Computer Interaction (ACI; e.g. [25, 45]), we are unaware of HCI research which seeks to promote engagement with nature and wildlife in this way (though see REF). ACI is typically concerned with animals that have existing relationships with humans, whether as pets [16], farm animals [11] or zoo inhabitants [12], and focuses on devices with which such creatures interact, often developed using some variation of 'pawticipatory' design [26]. Several of our early proposals (see Figure 2) were more interventionist and might benefit from ACI approaches and principles. My Naturewatch Cameras, however, are primarily intended to be ignored by animals, and to allow them to go about their business relatively undisturbed.

Instead, the purpose of My Naturewatch Cameras is simply to support people in being aware of the wildlife in their local area and to capture images of the creatures living nearby. Comments on our website and on social media suggest that

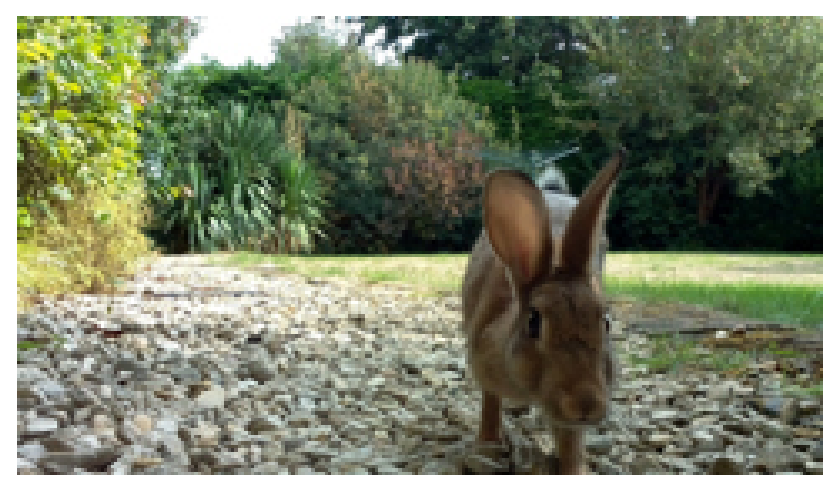

Figure 8: Carole's rabbit. 
peoples' engagement went beyond merely setting out the cameras with some bait, to include enquiries about what sorts of birds were in their gardens, where certain animals might be found, what they might eat and so forth. Moreover, the value people placed in viewing local birds and animals suggests that they started to recognize creatures as individuals, rather than, for instance, 'a bird' or even 'a magpie'-a recognition that Chris Packham sees as an invaluable motivator for conservation [Packham, personal communication].

My Naturewatch Cameras can thus be interpreted according to agendas such as familiarising wildlife, promoting conservation or ameliorating 'nature deficiency disorder' [24]. In our view, however, their primary value to users is simpler and perhaps more profound. In his essay Why Look At Animals?, the art critic John Berger wrote:

With their parallel lives, animals offer man a companionship which is different from any offered by human exchange. Different because it is a companionship offered to the loneliness of man as a species. ([3] p. 15)

In keeping with Berger, we suggest that the primary value of My Naturewatch Cameras is in allowing glimpses of the 'parallel lives' of the animals who are our neighbours. Moreover, we suggest that the intimacy of these encounters is heightened by the fact that the cameras are not well finished, commercial products, but DIY research products that people make themselves, using materials they have to hand, and close to the places they will be deployed.

\section{Engagement with Digital Making}

DIY research products depend to a large degree on advances in digital making. Centred on technology-oriented DIY activities, digital making has gained impetus with the increasing availability of low-cost microprocessor-based platforms such as Arduino and Raspberry $\mathrm{Pi}$ as well as affordable 3D printers and lasercutters. There is also a cultural and infrastructural dimension to the movement's growth, with events such as Maker Faires and Hackathons, physical resources such as FabLabs and London's Makerversity (makerversity.org/london/), code repositories such as GitHub, and crowdfunding sites such as Kickstarter all supporting communities of practice in the area.

Digital making, and maker culture more generally, has been a topic of much interest in the HCI community, with research variously providing overviews of existing communities and activities (e.g., [20, 21, 33, 38, 42]) and examples of products designed for open source making [7, 28, 29, 39, 40]. While much of this work celebrates making as an alternative to, or alternative route into, traditional commercial design (e.g., [20, 22, 23, 41]), others have identified impediments in the form of commercial disincentives [1] and, more broadly, limited access [19, 36]]. For instance, while making has been lauded as allowing disabled people to craft their own assistive technologies $[17,18]$, limits in making skills impedes the realization of this vision. A common approach to trying to increase accessibility is to run workshops supporting people to learn about digital making (e.g. [27], cf [18]). Questions remain, however, around the scalability of workshops and the likelihood that attendants will continue to make things without the scaffolding workshops afford.

My Naturewatch contributes to this literature by providing a case study of a DIY design that has motivated largescale involvement in digital making, including by people with little or no previous technical experience. Moreover, we have demonstrated that this can be achieved without direct contact, i.e. without workshops or maker spaces, through a combination of large scale publicity, a carefully crafted website, and an easy to make design.

Our experience suggests that key to this success has been the reduction or removal of impediments to making. All the components for My Naturewatch Camera are readily available online. The finished device is affordable. We work to make the instructions as clear as we can. We don't require people to register or make multiple visits to the website.

The resulting design is very simple, yet exposes its makers to many facets of digital design. Clipping a video cable into the Raspberry Pi may not be demanding, but it demonstrates the construction of devices from components. Downloading software emphasises its importance in the functionality of electronic devices, while editing a configuration file gives a taste of modifying code. Building housing from food storage containers promotes engagement with product design, and potentially considerations of aesthetics and cultural connotations. At each step, there are openings for further engagement, and these have been explored by people making the cameras. Overall, then, we suggest that My Naturewatch is an effective and accessible introduction to digital making. Moreover, we see the project as a promising first step in harnessing digital making and DIY designs in circulating research products, as we discuss next.

\section{DIY Design and Dissemination}

Practice research is well established in HCI and broader design communities (e.g. [14, 46]). As Pierce [34] has pointed out, however, few people have first-hand experience with physically-realised 'research products' [32]. In many cases, only one or a few examples of a given design are produced, which means they are usually encountered via images in articles, lectures, online or, more rarely, in exhibitions. While articulation is important for design research $[6,14]$, such limited channels of dissemination prevent many from gaining the embodied understanding that comes from experience 
[37]. This raises the question: Given that few design products are distributed commercially, whether because their creators are uninterested or unsuccessful in taking them to market, how can we develop alternative pathways for their dissemination?

In previous projects, some researchers have turned to batch production to increase the number of people who can try the research products they create, both to permit large-scale field studies and to disseminate the ideas they embody [5, 15, 31, 35]. Batch production, however, is costly and time-consuming, and for digital devices typically remains quite limited in the numbers of devices that can be made-for instance, even the large scale Datacatcher project only produced 130 devices [15].

Thus, recent research has begun to explore DIY products as a method for disseminating practice research (e.g., [10]). For instance, the Interaction Research Studio has made available designs and custom components, sold at cost, for cameras and audio devices specialized for Cultural Probe studies [5]. In addition to a means for the designs themselves to be disseminated, the idea is that DIY making can also allow the ideas such designs embody to reach varied audiences.

This project builds on that work, demonstrating how a research product can be experienced by over 1000 people by motivating them to build it themselves. This is a cause for optimism about the prospects for DIY design as a means for circulating design research-but also a cautionary tale about the challenges involved. First, given that approximately 1,500 people viewed Springwatch for every one who have made a camera, it seems clear that successful releases of future DIY design products will require similarly large-scale, or much better targeted, public exposure. Moreover, My Naturewatch Camera seems to have succeeded because of its simplicity and clarity of use: persuading people to make their own speculative, critical or counterfactual designs is likely to be more difficult [10]. For designers seeking to critique existing trends, extrapolate future possibilities, or study unusual design premises or forms of engagement, DIY design may be unsuitable. For those researchers exploring alternatives to prevailing trends in technology, however, producing designs that simultaneously embrace new values and are broadly appealing can be a useful and even necessary challenge.

Finally, minimising barriers to involvement means we forego deeper engagement with our makers: from this point of view we know little about the majority of people who are making the cameras. In future studies, this could be avoided by requiring people to register their details before gaining access to plans for building. Anybody willing to copy and republish the making instructions could easily subvert this, however-and indeed, Pimoroni has republished a PDF version of the My Naturewatch instructions without asking our permission. Preventing shadow sites and ensuring registration could be achieved by requiring essential components of given DIY research products to be obtained from our research group, but this would seem inevitably to reduce the circulation of our research projects. Moreover, there is an appeal to us in having close access to a subset of users, whether satisfied or annoyed, and allowing others to be known only through occasional social media posts or to evade scrutiny entirely. This recreates the logic of traditional sample-based research while providing ample material for research insights-and we find it pleasing to think of a large number of people enjoying My Naturewatch Cameras without interference.

In sum, the fact that so many people have made $\mathrm{My} \mathrm{Na-}$ turewatch Cameras shows that, with appropriate design and adequate publicity, research products can circulate widely outside commercial markets. Challenges remain for developing DIY design as a research methodology. For us, nonetheless, this is an encouraging foundation on which to build further research.

\section{CONCLUSION}

This paper contributes the design for a self-build wildlife camera offering offers several advantages over current camera traps that are available commercially or from other research projects. First, it uses computer vision for motion detection, which reduces the false positives caused by infrared motion detection. Second, it includes a smartphone interface which allows easy set-up and retrieval of images. Third, it is easy and inexpensive to build and customise using off-the-shelf components including household items. Finally, My Naturewatch Camera is designed to support open-ended engagement with local wildlife rather than utilitarian tracking, and produces images that are intimate and aesthetically pleasing.

In addition, the paper contributes a description of our strategy to engage people in making this computational product via an orchestrated effort involving a national primetime TV show, instructional website, and social media outreach. These contributions are mutually dependent: we designed the camera with Springwatch in mind, and our engagement efforts were successful because My Naturewatch Camera is suitable for that audience. Taken together, our methodological contribution is to highlight that the co-development of a research device and engagement strategy allows research products to be circulated at scale. Thus we see our primary research contribution as the description of the overall configuration of the project, including the relationship we forged with the Natural History Unit.

Our evidence suggests that our engagement strategy was successful insofar as over 1000 people were motivated to build My Naturewatch Cameras. We have had unsolicited 
feedback from hundreds of makers, and their comments (all publicly available on mynaturewatch.net/forum, pimoroni.com and Twitter ${ }^{\mathrm{TM}}$ ) suggest that most enjoyed building and using the cameras. Moreover, for many of them, the experience has had impact on their engagement with digital making and with local wildlife as they have become more aware and concerned with the various birds and animals they photograph. Perhaps our most significant contribution, then, is to all the people who have used the cameras to find new companionship with the creatures who live amongst us-and to the animals who will benefit from greater care as a consequence.

\section{ACKNOWLEDGEMENTS}

Many thanks to Chris Howard, Chris Packham, Colin Burns, Billy Clarke, Rosemary Edwards, Ailish Heneberry, Laura Howard and Sophie Meyjes from the BBC, to Jamie Dunning for expert ornithological advice, to Anne Schlottmann for comments and to all the people who made My Naturewatch Cameras. This research was supported by award EP/P006256/1 from the UK Engineering and Physical Sciences Research Council.

\section{REFERENCES}

[1] Morgan G. Ames, Jeffrey Bardzell, Shaowen Bardzell, Silvia Lindtner, David A. Mellis, and Daniela K. Rosner. 2014. Making Cultures: Empowerment, Participation, and Democracy - or Not?. In Proceedings of the Extended Abstracts of the 32Nd Annual ACM Conference on Human Factors in Computing Systems (CHI EA '14). ACM, New York, NY, USA, 1087-1092. https://doi.org/10.1145/2559206.2579405

[2] BBC. 2018. Blue Planet II. Retrieved 18 September 2018 from https: //www.bbc.co.uk/programmes/p04tjbtx

[3] John Berger. 2009. Why look at animals? Penguin Books. (book).

[4] Broadcasters' Audience Research Board. 2018. Weekly top 30 programmes. Retrieved 17 September 2018 from https://www.barb.co. uk/viewing-data/weekly-top-30/

[5] Andy Boucher, Dean Brown, Liliana Ovalle, Andy Sheen, Mike Vanis, William Odom, Doenja Oogjes, and William Gaver. 2018. TaskCam: Designing and Testing an Open Tool for Cultural Probes Studies. In Proceedings of the 2018 CHI Conference on Human Factors in Computing Systems (CHI '18). ACM, New York, NY, USA, Article 71, 12 pages. https://doi.org/10.1145/3173574.3173645

[6] John Bowers. 2012. The Logic of Annotated Portfolios: Communicating the Value of 'Research Through Design'. In Proceedings of the Designing Interactive Systems Conference (DIS '12). ACM, New York, NY, USA, 68-77. https://doi.org/10.1145/2317956.2317968

[7] Damien Brun, Susan M. Ferreira, Charles Gouin-Vallerand, and Sébastien George. 2016. CARTON Project: Do-It-Yourself Approach to Turn a Smartphone into a Smart Eyewear. In Proceedings of the 14th International Conference on Advances in Mobile Computing and Multi Media (MoMM '16). ACM, New York, NY, USA, 128-136. https: //doi.org/10.1145/3007120.3007134

[8] Discovery Channel. 2018. Shark Week. Retrieved 18 September 2018 from www.discoveryuk.com/shows/sharkweek/shark-week-2018

[9] Matthew A. Dalton, Audrey Desjardins, and Ron Wakkary. 2014. From DIY Tutorials to DIY Recipes. In CHI '14 Extended Abstracts on Human Factors in Computing Systems (CHI EA '14). ACM, New York, NY, USA, 1405-1410. https://doi.org/10.1145/2559206.2581238
[10] Audrey Desjardins, Ron Wakkary, Will Odom, Henry Lin, and Markus Lorenz Schilling. 2017. Exploring DIY Tutorials As a Way to Disseminate Research Through Design. Interactions 24, 4 (June 2017), 78-82. https://doi.org/10.1145/3098319

[11] Jessica Katherine Frawley and Laurel Evelyn Dyson. 2014. Animal Personas: Acknowledging Non-human Stakeholders in Designing for Sustainable Food Systems. In Proceedings of the 26th Australian Computer-Human Interaction Conference on Designing Futures: The Future of Design (OzCHI '14). ACM, New York, NY, USA, 21-30. https://doi.org/10.1145/2686612.2686617

[12] Fiona French, Clara Mancini, and Helen Sharp. 2017. Exploring Research Through Design in Animal Computer Interaction. In Proceedings of the Fourth International Conference on Animal-Computer Interaction (ACI2017). ACM, New York, NY, USA, Article 2, 12 pages. https://doi.org/10.1145/3152130.3152147

[13] William Gaver. 2011. Making Spaces: How Design Workbooks Work. In Proceedings of the SIGCHI Conference on Human Factors in Computing Systems (CHI '11). ACM, New York, NY, USA, 1551-1560. https://doi. org/10.1145/1978942.1979169

[14] William Gaver. 2012. What Should We Expect from Research Through Design?. In Proceedings of the SIGCHI Conference on Human Factors in Computing Systems (CHI '12). ACM, New York, NY, USA, 937-946. https://doi.org/10.1145/2207676.2208538

[15] William Gaver, Andy Boucher, Nadine Jarvis, David Cameron, Mark Hauenstein, Sarah Pennington, John Bowers, James Pike, Robin Beitra, and Liliana Ovalle. 2016. The Datacatcher: Batch Deployment and Documentation of 130 Location-Aware, Mobile Devices That Put Sociopolitically-Relevant Big Data in People's Hands: Polyphonic Interpretation at Scale. In Proceedings of the 2016 CHI Conference on Human Factors in Computing Systems (CHI '16). ACM, New York, NY, USA, 1597-1607. https://doi.org/10.1145/2858036.2858472

[16] Ilyena Hirskyj-Douglas and Janet C. Read. 2014. Who Is Really In The Center Of Dog Computer Design?. In Proceedings of the 2014 Workshops on Advances in Computer Entertainment Conference (ACE '14 Workshops). ACM, New York, NY, USA, Article 2, 5 pages. https: //doi.org/10.1145/2693787.2693793

[17] Amy Hurst and Shaun Kane. 2013. Making "Making" Accessible. In Proceedings of the 12th International Conference on Interaction Design and Children (IDC '13). ACM, New York, NY, USA, 635-638. https: //doi.org/10.1145/2485760.2485883

[18] Amy Hurst and Jasmine Tobias. 2011. Empowering Individuals with Do-it-yourself Assistive Technology. In The Proceedings of the 13th International ACM SIGACCESS Conference on Computers and Accessibility (ASSETS '11). ACM, New York, NY, USA, 11-18. https: //doi.org/10.1145/2049536.2049541

[19] Tom Jenkins and Ian Bogost. 2015. Escaping the Sandbox: Making and Its Future. In Proceedings of the Ninth International Conference on Tangible, Embedded, and Embodied Interaction (TEI '15). ACM, New York, NY, USA, 29-32. https://doi.org/10.1145/2677199.2680558

[20] Stacey Kuznetsov and Eric Paulos. 2010. Rise of the Expert Amateur: DIY Projects, Communities, and Cultures. In Proceedings of the 6th Nordic Conference on Human-Computer Interaction: Extending Boundaries (NordiCHI '10). ACM, New York, NY, USA, 295-304. https://doi.org/10.1145/1868914.1868950

[21] Stacey Kuznetsov, Alex S. Taylor, Eric Paulos, Carl DiSalvo, and Tad Hirsch. 2012. (DIY)Biology and Opportunities for HCI. In Proceedings of the Designing Interactive Systems Conference (DIS '12). ACM, New York, NY, USA, 809-810. https://doi.org/10.1145/2317956.2318085

[22] Silvia Lindtner, Anna Greenspan, and David Li. 2015. Designed in Shenzhen: Shanzhai Manufacturing and Maker Entrepreneurs. Aarhus Series on Human Centered Computing 1, 1 (Oct. 2015), 12. https: //doi.org/10.7146/aahcc.v1i1.21265 
[23] Silvia Lindtner, Garnet D. Hertz, and Paul Dourish. 2014. Emerging Sites of HCI Innovation: Hackerspaces, Hardware Startups \&\#38; Incubators. In Proceedings of the SIGCHI Conference on Human Factors in Computing Systems (CHI '14). ACM, New York, NY, USA, 439-448. https://doi.org/10.1145/2556288.2557132

[24] Richard Louv. 2008. Last child in the woods: Saving our children from nature-deficit disorder. Algonquin books. (book).

[25] Clara Mancini. 2013. Animal-computer Interaction (ACI): Changing Perspective on HCI, Participation and Sustainability. In CHI '13 Extended Abstracts on Human Factors in Computing Systems (CHI EA '13). ACM, New York, NY, USA, 2227-2236. https://doi.org/10.1145/ 2468356.2468744

[26] Demi Mankoff, Anind Dey, Jennifer Mankoff, and Ken Mankoff. 2005. Supporting Interspecies Social Awareness: Using Peripheral Displays for Distributed Pack Awareness. In Proceedings of the 18th Annual ACM Symposium on User Interface Software and Technology (UIST '05). ACM, New York, NY, USA, 253-258. https://doi.org/10.1145/1095034.1095076

[27] Janis Lena Meissner, John Vines, Janice McLaughlin, Thomas Nappey, Jekaterina Maksimova, and Peter Wright. 2017. Do-It-Yourself Empowerment As Experienced by Novice Makers with Disabilities. In Proceedings of the 2017 Conference on Designing Interactive Systems (DIS '17). ACM, New York, NY, USA, 1053-1065. https://doi.org/10. 1145/3064663.3064674

[28] David A. Mellis and Leah Buechley. 2014. Do-it-yourself Cellphones: An Investigation into the Possibilities and Limits of High-tech Diy. In Proceedings of the SIGCHI Conference on Human Factors in Computing Systems (CHI '14). ACM, New York, NY, USA, 1723-1732. https://doi. org $/ 10.1145 / 2556288.2557309$

[29] David A. Mellis, Dana Gordon, and Leah Buechley. 2011. Fab FM: The Design, Making, and Modification of an Open-source Electronic Product. In Proceedings of the Fifth International Conference on Tangible, Embedded, and Embodied Interaction (TEI '11). ACM, New York, NY, USA, 81-84. https://doi.org/10.1145/1935701.1935718

[30] Naturebytes. 2018. Digital making for wildlife. Retrieved 29 December 2018 from http://naturebytes.org

[31] William Odom, Ron Wakkary, Ishac Bertran, Matthew Harkness, Garnet Hertz, Jeroen Hol, Henry Lin, Bram Naus, Perry Tan, and Pepijn Verburg. 2018. Attending to Slowness and Temporality with Olly and Slow Game: A Design Inquiry Into Supporting LongerTerm Relations with Everyday Computational Objects. In Proceedings of the 2018 CHI Conference on Human Factors in Computing Systems (CHI '18). ACM, New York, NY, USA, Article 77, 13 pages. https://doi.org/10.1145/3173574.3173651

[32] William Odom, Ron Wakkary, Youn-kyung Lim, Audrey Desjardins, Bart Hengeveld, and Richard Banks. 2016. From Research Prototype to Research Product. In Proceedings of the 2016 CHI Conference on Human Factors in Computing Systems (CHI '16). ACM, New York, NY, USA, 2549-2561. https://doi.org/10.1145/2858036.2858447

[33] Aisling Ann O'Kane, Amy Hurst, Gerrit Niezen, Nicolai Marquardt, Jon Bird, and Gregory Abowd. 2016. Advances in DIY Health and Wellbeing. In Proceedings of the 2016 CHI Conference Extended Abstracts on Human Factors in Computing Systems (CHI EA '16). ACM, New York, NY, USA, 3453-3460. https://doi.org/10.1145/2851581.2856467
[34] James Pierce. 2014. On the Presentation and Production of Design Research Artifacts in HCI. In Proceedings of the 2014 Conference on Designing Interactive Systems (DIS '14). ACM, New York, NY, USA, 735-744. https://doi.org/10.1145/2598510.2598525

[35] James Pierce and Eric Paulos. 2015. Making Multiple Uses of the Obscura 1C Digital Camera: Reflecting on the Design, Production, Packaging and Distribution of a Counterfunctional Device. In Proceedings of the 33rd Annual ACM Conference on Human Factors in Computing Systems (CHI '15). ACM, New York, NY, USA, 2103-2112. https://doi.org/10.1145/2702123.2702405

[36] Oliver Quinlan. 2015. Young Digital Makers: Surveying attitudes and opportunities for digital creativity across the UK. Nesta. (book).

[37] Johan RedstrÃüm. 2017. Making design theory. MIT Press. (book).

[38] Daniela Rosner and Jonathan Bean. 2009. Learning from IKEA Hacking: I'm Not One to Decoupage a Tabletop and Call It a Day.. In Proceedings of the SIGCHI Conference on Human Factors in Computing Systems (CHI '09). ACM, New York, NY, USA, 419-422. https://doi.org/10.1145/ 1518701.1518768

[39] Daniel Saakes. 2009. Big Lampan Lamps: Designing for DIY. In Proceedings of the Seventh ACM Conference on Creativity and Cognition (C\&\#38;C '09). ACM, New York, NY, USA, 403-404. https: //doi.org/10.1145/1640233.1640322

[40] Greg Saul, Cheng Xu, and Mark D. Gross. 2010. Interactive Paper Devices: End-user Design \&\#38; Fabrication. In Proceedings of the Fourth International Conference on Tangible, Embedded, and Embodied Interaction (TEI '10). ACM, New York, NY, USA, 205-212. https://doi. org/10.1145/1709886.1709924

[41] Joshua G. Tanenbaum, Amanda M. Williams, Audrey Desjardins, and Karen Tanenbaum. 2013. Democratizing Technology: Pleasure, Utility and Expressiveness in DIY and Maker Practice. In Proceedings of the SIGCHI Conference on Human Factors in Computing Systems (CHI '13). ACM, New York, NY, USA, 2603-2612. https://doi.org/10.1145/2470654. 2481360

[42] Tiffany Tseng and Mitchel Resnick. 2014. Product Versus Process: Representing and Appropriating DIY Projects Online. In Proceedings of the 2014 Conference on Designing Interactive Systems (DIS '14). ACM, New York, NY, USA, 425-428. https://doi.org/10.1145/2598510.2598540

[43] Daniel Weil. 1981. Radio in a Bag. Retrieved 21 September 2018 from https://collections.vam.ac.uk/item/O85208/ radio-in-abag-radio-weil-daniel/

[44] Wikipedia. 2018. Camera Trap. Retrieved 17 September 2018 from https://en.wikipedia.org/wiki/Camera_trap

[45] Anna Zamansky, Amanda Roshier, Clara Mancini, Emily C. Collins, Carol Hall, Katie Grillaert, Ann Morrison, Steve North, and Hanna Wirman. 2017. A Report on the First International Workshop on Research Methods in Animal-Computer Interaction. In Proceedings of the 2017 CHI Conference Extended Abstracts on Human Factors in Computing Systems (CHI EA '17). ACM, New York, NY, USA, 806-815. https://doi.org/10.1145/3027063.3052759

[46] John Zimmerman, Jodi Forlizzi, and Shelley Evenson. 2007. Research Through Design As a Method for Interaction Design Research in HCI. In Proceedings of the SIGCHI Conference on Human Factors in Computing Systems (CHI '07). ACM, New York, NY, USA, 493-502. https://doi. org/10.1145/1240624.1240704 